\title{
Decreased activity of butyrylcholinesterase in blood plasma of patients with chronic obstructive pulmonary disease
}

\author{
Paulina Sicinska ${ }^{1}$, Bozena Bukowska ${ }^{1}$, Aneta Pajak², Aneta Koceva-Chyla ${ }^{3}$, Tadeusz Pietras ${ }^{2}$, \\ Piotr Nizinkowski ${ }^{1}$, Paweł Gorski², Maria Koter-Michalak ${ }^{1}$
}

\begin{abstract}
1Department of Environmental Pollution Biophysics, Faculty of Biology and Environmental Protection, University of Lodz, Lodz, Poland

${ }^{2}$ Department of Pneumology and Allergology, Norbert Barlicki Memorial University Hospital, Medical University of Lodz, Lodz, Poland

${ }^{3}$ Department of Thermobiology, Faculty of Biology and Environmental Protection, University of Lodz, Lodz, Poland
\end{abstract}

Submitted: 12 June 2015

Accepted: 27 August 2015

Arch Med Sci 2017; 13, 3: 645-651

DOI: 10.5114/aoms.2016.60760

Copyright @ 2016 Termedia \& Banach

\author{
Corresponding author: \\ Paulina Sicinska \\ Department of Environmental \\ Pollution Biophysics \\ Faculty of Biology and \\ Environmental Protection \\ University of Lodz \\ 141/143 Pomorska St \\ 90-236 Lodz, Poland \\ Phone: +48 426354453 \\ Fax: +48 426354473 \\ E-mail:sicinskp@ \\ biol.uni.lodz.pl
}

\begin{abstract}
Introduction: Butyrylcholinesterase (BChE) is involved in the metabolism of endogenous lipids and xenobiotics, such as esters of carboxylic or phosphoric acids. Butyrylcholinesterase activity is associated with both inflammation and oxidative stress. Changes in the activity of this enzyme have been observed in various diseases such as liver cirrhosis, diabetes, neurodegenerative disease and others.

Material and methods: The study involved 30 patients with chronic obstructive pulmonary disease (COPD) and 18 healthy subjects. The COPD patients were divided according to the severity of the disease by applying the classification of COPD based on GOLD standards for forced expiratory volume in $1 \mathrm{~s}$

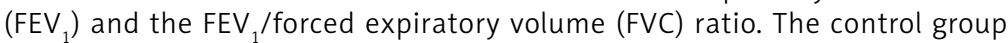
comprised blood samples collected from healthy subjects without concomitant diseases related to the respiratory system. Butyrylcholinesterase activity, lipid peroxidation and total antioxidant capacity (TAC) were determined in the blood plasma.

Results: A significant $(p<0.05)$ decrease in the activity of BChE, associated with an increase in lipid peroxidation and a decrease in the total antioxidant capacity, was observed in blood plasma of patients with chronic obstructive pulmonary disease.

Conclusions: The study shows for the first time that activity of BChE in the blood plasma of patients diagnosed with chronic obstructive pulmonary disease is considerably reduced compared with healthy subjects. These changes were accompanied by a decrease of TAC and an increase of lipid peroxidation, which suggests that they may be related to the oxidative stress induced by COPD disease.
\end{abstract}

Key words: butyrylcholinesterase, chronic obstructive pulmonary disease, pulmonary disease, lipid peroxidation, total antioxidant capacity, oxidative stress. 


\section{Introduction}

Acetylcholinesterase (AChE) and butyrylcholinesterase (BChE) are cholinesterases, enzymes that catalyze the hydrolysis of the neurotransmitter acetylcholine into choline and acetic acid, an essential reaction enabling the return of the activated cholinergic neuron to the resting state. The two types of cholinesterases: AChE and BChE, have different preferences for the substrates acetylcholine and butyrylcholine, respectively. Acetylcholinesterase, also called red blood cell cholinesterase, is more abundant in the erythrocyte membranes, central nervous system and skeletal muscle, whereas BChE, also called plasma cholinesterase, is secreted into plasma and synthesized in the liver [1].

Human blood plasma contains almost exclusively BChE, whereas animal blood plasma contains variable proportions of both $\mathrm{BChE}$ and AChE. The exact physiological function of BChE remains elusive, although it could act as an alternative to the homologous AChE as a scavenger for anticholinesterase compounds [2]. Despite being located in different places, the enzymes are able to cooperate. Butyrylcholinesterase can hydrolyze both butyrylcholine and acetylcholine esters and consequently regulate the acetylcholine level $[3,4]$.

Due to its esterase activity, BChE plays an important role in hydrolysis of numerous lipids, drugs and xenobiotics before they reach AChE. Butyrylcholinesterase hydrolyzes (detoxifies) hydrophilic and hydrophobic compounds containing esters of carboxyl or phosphoric acids; for that reason $B C h E$ is classified as a detoxifying ester, important from the pharmacological point of view [5]. Butyrylcholinesterase is also involved in the hydrolysis of hormones as exemplified by the degradation of ghrelin, a hormone responsible for the feeling of hunger. Ghrelin is inactivated by hydrolysis of the ester bond by BChE in the human blood plasma. In this case the enzyme acts as a hydrolase and converts ghrelin to inactive desacyl ghrelin [6]. This reaction results in a reduction of the feeling of hunger and a decrease of aggression, as has been demonstrated in studies on rats [7].

Chronic obstructive pulmonary disease (COPD) is a disease characterized mostly by the restricted flow of air through the airways, and an improper lung inflammatory response to harmful dust and gas. The COPD is associated with increased risk of respiratory infections, lung cancer, pulmonary embolism, pneumothorax, ischemic heart disease, diabetes and depression [8, 9]. A characteristic feature of the disease is its constant progression. The airflow cannot be restored to the initial value. An appropriate treatment is aimed only at slowing the process down [10-12].
Presently, research is underway on COPD therapy in the following areas: the new inhaled $\beta$-agonist and cholinolytic [13], inhibitors of phosphodiesterase [14] and additional treatment of comorbidity.

Tobacco smoking is the principal cause of the disease $[15,16]$. Other risk factors of COPD are: industrial pollution (e.g. coal and silica dust inhaled by workers, cotton dust or grain dust) and chemicals (e.g. herbicides and pesticides) [17, 18]. Studies demonstrate that farmers exposed to pesticide aerosols are at a significant risk of problems with pulmonary respiratory sufficiency, and therefore of COPD. Another type of pollution that may induce COPD is domestic pollution emerging from burning wood, animal feces, vegetable residues and coal in furnaces poorly adapted to the task [19].

Exposure to these factors leads to an increased amount of reactive oxygen and nitrogen species released from leukocytes and macrophages, occurring in COPD patients and associated with inflammatory processes [20-22] and allergic inflammation [23]. Disruption of the pro- and antioxidative equilibrium may cause an increased pulmonary inflammatory reaction, leading to elevated expression of tumor necrosis factor (TNF- $\alpha$ ), interleukin (IL)-6, IL-8, and IL-1 $\beta$, which are used as diagnostic markers in that disease [24, 25]. Human BChE and paraoxonase- 1 are two major bioscavenger enzymes that are associated with inflammation, oxidative stress and lipid metabolism [26]. Moreover, due to its properties, BChE participates in the metabolism of numerous xenobiotics [27-29] and drugs, including those used for COPD treatment, such as aclidinium bromide [30]. Hence, oxidative stress may modify the activity of the enzyme [31].

There are currently no specific studies indicating the mechanism of action of BChE in COPD patients. Acetylcholine is the main neurotransmitter of the parasympathetic system. In the respiratory tract the activity of AChE and BChE is particularly important in COPD, where they may be associated with the decomposition of acetylcholine and regulation of its level.

This, in turn, provides regulation of bronchial channels narrowing and mucus secretion. BChE, as mentioned above, can also participate in the metabolism of drugs used for COPD treatment, and accordingly alterations of its activity may influence the efficacy of the therapy $[3,4]$.

The aim of the present study was to determine whether there are any alterations in activity of $\mathrm{BChE}$ in the blood plasma of COPD patients and, if so, how these changes are related to the redox state (total antioxidant capacity and plasma lipid peroxidation) of plasma. 


\section{Material and methods}

\section{Chemicals}

5.5'-dithio-bis-2-nitrobenzoic acid (DTNB) and butyrylthiocholine iodide (BTC) were from Sigma Switzerland; trichloroacetic acid (TCA) and thiobarbituric acid (TBA) were provided by Sigma Germany, 2,2'-azino-bis(3-ethylbenzothiazoline-6-sulfonic acid) diammonium salt (ABTS) and 6-hydroxy-2,5,7,8-tetramethylchromane-2-carboxylic acid (Trolox) were purchased from Sigma USA.

\section{Study population}

The investigated group consisted of 30 COPD patients aged $66 \pm 8$ diagnosed with stage I or II in the Department of Pneumology and Allergology of Norbert Barlicki Memorial University Hospital in Lodz, Poland.

The COPD patients were divided according to the severity of the disease by applying the classification of COPD based on GOLD standards for forced expiratory volume in $1 \mathrm{~s}\left(\mathrm{FEV}_{1}\right)$ and $\mathrm{FEV}_{1} /$ forced expiratory volume (FVC) ratio. The $\mathrm{FEV}_{1} /$ FVC ratio was determined, and was below the lower limit of normal $\left(\mathrm{FEV}_{1} / \mathrm{FVC}=72.6 \pm 1.6 \%\right)$. Arterial oxygen pressure was $59 \pm 14 \mathrm{~mm} \mathrm{Hg}$ and arterial carbon dioxide pressure was $41 \pm 10 \mathrm{~mm} \mathrm{Hg}$. Patients were not subjected to any home/hospital oxygen treatment although they were qualified for such therapy. All patients were in a stable stage of the disease and they did not experience any infectious exacerbations during this time. The investigated group consisted of patients with COPD newly diagnosed following treatment.

The control group, aged $60 \pm 10$, comprised 18 blood samples collected from healthy subjects without concomitant diseases related to the respiratory system. In the control group, the correct values were adopted by the standards for the diagnostic laboratory performing the appropriate tests.

The testing was approved by the Bioethics Committee of the Medical University of Lodz. (No. RNN/316/13/KE z 17.12.2013). The testing was approved by the Bioethics Committee of the Medical University of Lodz. (No. RNN/316/13/KE dated 17.12.2013).

\section{Plasma collection}

Plasma samples were collected from both patients and healthy persons (controls). Peripheral blood was collected into tubes with anticoagulant (sodium citrate) and then centrifuged at $600 \mathrm{~g}$ for $10 \mathrm{~min}$ at $4^{\circ} \mathrm{C}$. After centrifugation, plasma was collected and analyzed for the selected parameters.

\section{Butyrylcholinesterase activity}

The activity of plasma butyrylcholinesterase (BChE; E.C. 3.1.1.8) was determined using butyr- ylthiocholine iodide (BTC) as a substrate. Assays were carried out in $50 \mathrm{mM}$ phosphate buffer, $\mathrm{pH}$ 8.0 , at $25^{\circ} \mathrm{C}$. Eight $\mu \mathrm{l}$ of total blood plasma was mixed with $2 \mathrm{ml}$ of phosphoric buffer $(\mathrm{pH} 8.0)$. Then $20 \mu \mathrm{l}$ of $10 \mathrm{mM}$ DTNB and $80 \mu \mathrm{l}$ of $0.4 \mathrm{mM}$ of BTC were added to the samples and the absorbance was measured at $412 \mathrm{~nm}$ for $1 \mathrm{~min}$. BChE activity was estimated from the slope of the $a b$ sorbance curve. One unit of BChE activity was defined as the amount of $\mu$ moles of butyrylthiocholine degraded for $1 \mathrm{~min}$ by BChE contained in $1 \mathrm{ml}$ of the blood plasma [32].

\section{Peroxidation of lipids}

Lipid peroxidation in plasma was quantified by measuring the formation of thiobarbituric acid reactive substances (TBARS). $1 \mathrm{ml}$ of blood plasma was mixed with $20 \%$ trichloroacetic acid (TCA) $(1: 1)$. Samples were centrifuged at $600 \mathrm{~g}$ for $10 \mathrm{~min}$. Thiobarbituric acid (TBA) was added to the supernatant and samples were heated at $100^{\circ} \mathrm{C}$ for $15 \mathrm{~min}$. The absorbance of the cooled supernatant was measured at $532 \mathrm{~nm}$ [33]. Lipid peroxidation was expressed in absorbance units.

\section{Total plasma antioxidant capacity}

Total plasma antioxidant capacity was determined by means of reduction of ABTS. Absorbance measurement was performed at $414 \mathrm{~nm}$. Trichloroacetic acid was expressed in one-electron equivalents of Trolox. Considering that one Trolox molecule reacts with two $\mathrm{ABTS}^{+}$molecules, the calculated values were multiplied by two (unit per $\mu \mathrm{mol}$ of Trolox equivalent $\mathrm{I}^{-1}$ ) [34].

\section{Statistical analysis}

The results are presented as mean \pm SD. Differences between groups were assessed by an unpaired Student's $t$ test. The statistical analysis was performed using Statistica, version 9.0 PL software (StatSoft Inc., Tulsa, USA). Each sample from a patient with COPD and controls was measured in 3 replicates for each method.

\section{Results}

\section{Butyrylcholinesterase activity}

Butyrylcholinesterase activity in COPD patients was significantly reduced $(0.93 \pm 0.45 \mu \mathrm{mol} / \mathrm{min} /$ $\mathrm{ml}$ P.C.) compared with the control group (1.43 $\pm 0.43 \mu \mathrm{mol} / \mathrm{min} / \mathrm{ml} \mathrm{P.C.)}, p<0.01$ (Figure 1 ).

\section{Lipid peroxidation}

Lipid peroxidation in the blood plasma of COPD patients was significantly $(p<0.05)$ more extensive than in healthy individuals. The values are 


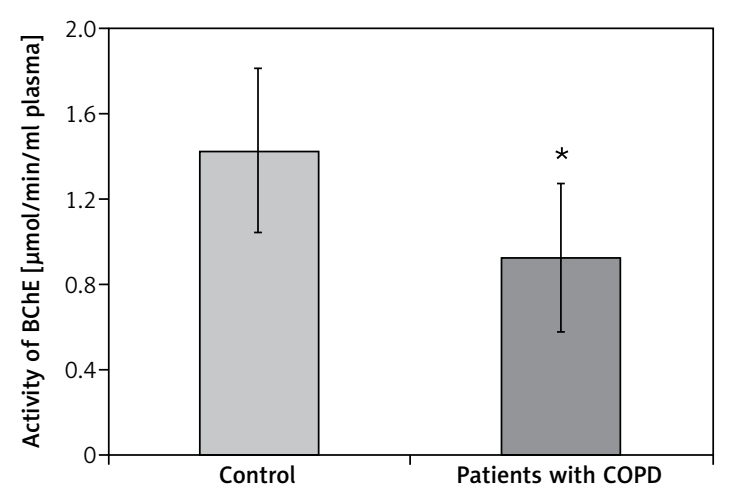

Figure 1. BChE activity in healthy plasma donors and patients with COPD $\left({ }^{*} p<0.01\right)$

$0.028 \pm 0.005$ arbitrary units $/ \mathrm{ml}$ plasma in COPD patients and $0.018 \pm 0.004$ arbitrary units $/ \mathrm{ml}$ plasma in the control group (Figure 2).

\section{Total plasma antioxidant capacity}

Total plasma antioxidant capacity assay confirmed impaired redox state and weakened antioxidant defense of blood plasma of COPD patients compared with healthy subjects. TAC of COPD blood plasma was significantly lower $(p<0.05)$ than TAC of the healthy control group: $294 \pm 28 \mathrm{mmol}$ eqT/l and $340 \pm 20 \mathrm{mmol}$ eqT/l, respectively (Figure 3 ).

\section{Discussion}

Changes in BChE activity are observed in various diseases and poor health condition [35, 36]. Reduced plasma cholinesterase activity was reported in diseases associated with injury of the hepatic parenchyma $[37,38]$ such as liver fibrosis, hepatitis and cirrhosis [39]. Individuals with confirmed liver cirrhosis present a 5-fold lower level of BChE than healthy controls [1]. It has been found that the fall of enzyme activity is more pronounced in liver cirrhosis patients than in hepatitis patients [38]. Moreover, cholinesterases have been considered as a useful marker of the effect of primary malnutrition on hepatic function [39].

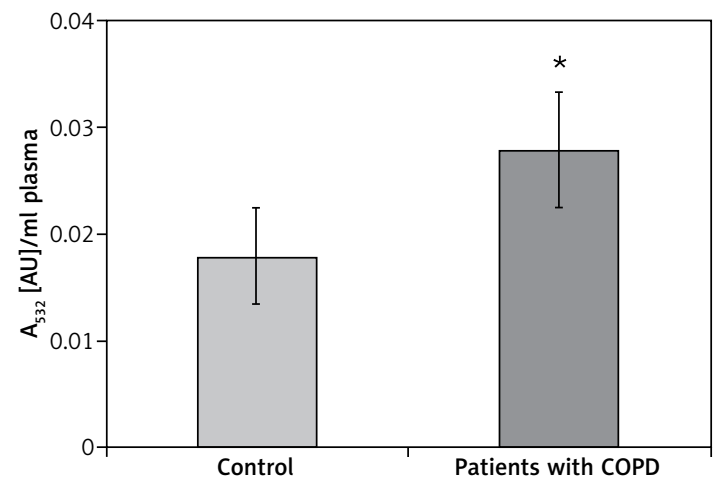

Figure 2. Lipid peroxidation in healthy plasma donors and patients with COPD $\left({ }^{*} p<0.05\right)$
An increase in the activity of BChE with increasing body mass index, activity of alanine aminotransferase, $\gamma$-glutamyltransferase, triglyceride level and a decreases in enzyme activity with in creasing high-density lipoprotein cholesterol have been reported for type 2 Japanese diabetic patients. Furthermore, a correlation between the in crease in BChE activity and the insulin resistance has been found, as well [40, 41].

In neurodegenerative diseases, the loss of cholinergic neurons leads to a significant decrease in the activity of brain AChE and a rapid increase in the BChE activity [4]. A similar tendency is observed with age: AChE activity decreases and BChE activity increases [42].

This study focuses on the assessment of plasma BChE activity in patients with COPD. A significant $(35 \%)$ reduction $(p<0.01)$ of the enzyme activity was demonstrated in COPD patients compared with healthy individuals (Figure 1). An increased amount of reactive oxygen species (ROS) has been reported in COPD patients. The ROS are released from inflammatory cells or airways during ventilation, or as a result of action of xenobiotics from, among other things, tobacco smoke [22]. An increased oxidative burden occurring in the lungs of COPD patients leads to disruption of the equilibrium between oxidants and the antioxidant defense system $[43,44]$.

Therefore, we studied the total antioxidative capacity and lipid peroxidation level, essential biomarkers of oxidative stress in the blood plasma of COPD patients [45]. The study demonstrated that in COPD patients the level of lipid peroxidation was elevated by $55 \%$ and antioxidative capacity was reduced by approximately $14 \%$ compared to healthy individuals (Figures 2 and 3).

Our data on lipid peroxidation and TAC are consistent with results reported by other authors [42, $44,46]$. Domej et al. observed an increased level of TBARS in the blood plasma of smokers with chronic bronchitis, compared to healthy non-smokers. Moreover, an increased level of secondary lipid

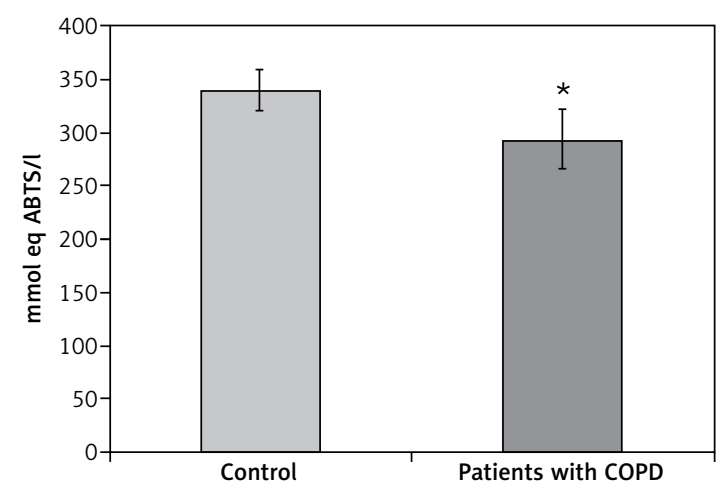

Figure 3. Total antioxidant capacity in healthy plasma donors and patients with COPD $\left({ }^{*} p<0.05\right)$ 
peroxidation products, including coupled dienes of linoleic acid, was also observed in these patients [42]. Gencer et al. observed a decrease in TAC level in the blood plasma of COPD patients [46]. Some earlier studies on smoke-exposed individuals demonstrated a reduction of the serum total antioxidant capacity compared to the controls $[22,47]$.

Increased lipid peroxidation may lead to altered activity of antioxidant enzymes. Waseem et al. observed lower activity of all analyzed antioxidant enzymes in serum of COPD smokers. A total of 60 controls (healthy non-smokers) and 121 smokers with COPD were studied. The authors observed a significant reduction in the antioxidant enzymes SOD, catalase and GPX in the smoker group as compared to healthy controls [48]. Our recent study indicated changes in the activity of antioxidant enzymes in COPD patients. We observed an increase in the activity of glutathione peroxidase and a decrease in the activity of superoxide dismutase, but not in the activity of catalase activity [49]. However, Nadeem et al., Gumral et al. and Woźniak et al. observed changes in activity of all three enzymes - CAT, GSH-PX and SOD [50-52].

It is well known that reactive oxygen species, such as hydrogen peroxide, affect the activity of cholinesterase. Schallreuter et al. reported that high concentrations of $\mathrm{H}_{2} \mathrm{O}_{2}$ inhibited acetylcholinesterase activity. The authors suggest that $\mathrm{H}_{2} \mathrm{O}_{2}$-mediated oxidation of amino acids at the active site of the enzyme could deactivate the protein [53].

Our results are in agreement with other studies demonstrating that decrease of human BChE activity is associated with oxidative stress [26, 54]. Tagliari et al. found that an increase in oxidative stress was associated with inhibition of BChE and an increase in homocysteine levels in blood of rats [21].

An increased lipid peroxidation level causes a reduction of the TAC [22]. That in turn may induce a reduction of $\mathrm{BChE}$ activity it seems that BChE activity changes in COPD are highly important, among other things because hydrolysis and activation of drugs used in the treatment of the disease depend on the activity of the esterase.

In conclusion, this study shows for the first time that the activity of butyrylcholinesterase in blood plasma of patients with COPD is significantly reduced compared with healthy persons, and that these changes are related to the oxidative stress associated with COPD disease and demonstrated by a decrease of TAC and an increase in lipid peroxidation in the blood plasma of COPD patients.

\section{Conflict of interest}

The authors declare no conflict of interest.

\section{References}

1. Ramachandran J, Sajith K, Priya S, Dutta A, Balasubramanian K. Serum cholinesterase is an excellent biomarker of liver cirrhosis. Trop Gastroenterol 2014; 35: 15-20.

2. Schwarz M, Glick D, Loewenstein Y, Soreq H. Engineering of human cholinesterase explains and predicts diverse cosequences of administration of various drugs and poisons. Pharmacol Ther 1999; 67: 283-322.

3. Shao Z. Comparison of the efficacy of four cholinesterase inhibitors in combination with memantine for the treatment of Alzheimer's disease. Int J Clin Exp Med 2015; 15: 2944-3294.

4. Bukowska B, Pieniążek D, Hutnik K, Duda W. Acetyloand butyrylocholinoesterase - structure, functions and its inhibitor. Curr Topics Biophys 2007; 30: 11-23.

5. Barata C, Solayan A, Porte C. Role of B-esterases in assessing toxicity of organophosphorus (chlorpyrifos, malathion) and carbamate (carbofuran) pesticides to Daphnia magna. Aquat Toxicol 2004; 10: 125-39.

6. Schopfer M, Lockridge O, Brimijoin S. Pure human butyrylcholinesterase hydrolyzes octanoyl ghrelin to desacyl ghrelin. Gen Comp Endocrinol 2015; 224: 61-8.

7. Chen V, Gao Y, Geng L, et al. Plasma butyrylcholinesterase regulates ghrelin to control aggression. Proc Natl Acad Sci U S A 2015; 17: 2251-6.

8. Kaźmierczak M, Ciebiada M, Pękala-Wojciechowska A, Pawłowski M, Nielepkowicz-Goździńska A, Antczak A. Evaluation of markers of inflammation and oxidative stress in COPD patients with or without cardiovascular comorbidities. Heart Lung Circ 2015; 24: 817-23.

9. Strelitz J, Engel L, Keifer M. Blood acetylcholinesterase and butyrylcholinesterase as biomarkers of cholinesterase depression among pesticide handlers. Occup Environ Med 2014; 71: 842-7.

10. Montoya-Estrada A, Torres-Ramos Y, Flores-Pliego A, et al. Urban, PM2.5 activates GAPDH and induces RBC damage in COPD patients. Front Biosci 2013; 5: 638-49.

11. Torres-Ramos Y, Montoya-Estrada A, Guzman-Grenfell A, et al. Urban PM2.5 induces ROS generation and RBC damage in COPD patients. Front Biosci 2011; 3: 808-17.

12. Chhabra S, Dash D. Acute exacerbations of chronic obstructive pulmonary disease: causes and impacts. Indian J Chest Dis Allied Sci 2014; 56: 93-104.

13. Rossi A, Zanardi E, Poletti V, Cazzola M. Clinical role of dual bronchodilation with an indacaterol-glycopyrronium combination in the management of COPD: its impact on patient-related outcomes and quality of life. Int J Chron Obstruct Pulmon Dis 2015; 10: 1383-92.

14. Page CP. Phosphodiesterase inhibitors for the treatment of asthma and chronic obstructive pulmonary disease. Int Arch Allergy Immunol 2014; 165: 152-64.

15. Liu Y, Pleasants R, Croft J, et al. Smoking duration, respiratory symptoms, and COPD in adults aged $\geq 45$ years with a smoking history. Int J Chron Obstruct Pulmon Dis 2015; 10: 1409-16.

16. Kiljander T, Helin T, Venho K, Jaakkola A, Lehtimäki L. Prevalence of asthma-COPD overlap syndrome among primary care asthmatics with a smoking history: a cross-sectional study. NPJ Prim Care Respir Med 2015; 25: 15047.

17. Johnston F, Purdie S, Jalaludin B, et al. Air pollution events from forest fires and emergency department attendances in Sydney, Australia 1996-2007: a case-crossover analysis. Environ Health 2014; 13: 105. 
18. Tian L, Ho K, Wang T, et al. Ambient carbon monoxide and the risk of hospitalization due to chronic obstructive pulmonary disease. Am J Epidemiol 2014; 15: 1159-67.

19. Song Q, Christiani D, Xiaorong W, Ren J. The global contribution of outdoor air pollution to the incidence, prevalence, mortality and hospital admission for chronic obstructive pulmonary disease: a systematic review and meta-analysis. Int J Environ Res Public Health 2014; 14: 11822-32.

20. Langen R, Korn S, Wouters E. ROS in the local and systemic pathogenesis of COPD. Free Rad Biol Med 2003; 35: 226-35.

21. Tagliari B, dos Santos T, Cunha A, et al. Chronic variable stress induces oxidative stress and decreases butyrylcholinesterase activity in blood of rats. J Neural Transm 2010; 117: 1067-76.

22. Onyesom I, Ighodayenowho O, Nwoke E. Serum total anti-oxidant capacity of some Nigerian cigarette smokers. East Afr J Public Health 2011; 8: 224-5.

23. Tworek D, Kuna P, Młynarski W, Górski P, Pietras T, Antczak A. MIG (CXCL9), IP-10 (CXCL10) and I-TAC (CXCL11) concentrations after nasal allergen challenge in patients with allergic rhinitis. Arch Med Sci 2013; 9: 849-53.

24. MacNee W. Treatment of stable COPD: antioxidants. Eur Respir Rev 2005; 14: 12-22.

25. Shaw J, Vaughan A, Dent A, et al. Biomarkers of progression of chronic obstructive pulmonary disease (COPD). J Thorac Dis 2014; 6: 1532-47.

26. Bahrehmand F, Vaisi-Raygani A, Rahimi Z, et al. Synergistic effects of BuChE non-UU phenotype and paraoxonase (PON1) $55 \mathrm{M}$ allele on the risk of systemic lupus erythematosus: influence on lipid and lipoprotein metabolism and oxidative stress, preliminary report. Lupus 2014; 23: 263-72.

27. Bodur E, Cokugras A, Tezcan E. Inhibition effects of benactyzine and drofenine on human serum butyrylcholinesterase. Arch Biochem Biophys 2001; 386: 25-99.

28. Müller T, Rocha J, Morsch V, Neis R, Schetinger M. Antidepressants inhibit human acetylcholinesterase and butyrylcholinesterase activity. Biochim Biophys Acta 2002; 21: 92-8.

29. Chtourou Y, Fetoui H, Gdoura R. Protective effects of naringenin on iron-overload-induced cerebral cortex neurotoxicity correlated with oxidative stress. Biol Trace Elem Res 2014; 158: 376-83.

30. Albertí J, Martinet A, Sentellas S, Salva M. Identification of the human enzymes responsible for the enzymatic hydrolysis of aclidinium bromide. Drug Metabol Dispos 2010; 38: 1202-10.

31. Sales M. Cholinergic drugs as therapeutic tools in inflammatory diseases: participation of neuronal and non-neuronal cholinergic systems. Antiinflamm Antiallergy Agents Med Chem 2013; 12: 109-16.

32. Ellman G, Courtney K, Andres V, Featerstone R. A new and rapid colorimetric determination of acetylcholinesterase activity. Biochem Pharmacol 1961; 7: 88-95.

33. Stocks J, Dormandy T. The autoxidation of human red cell lipids induced by hydrogen peroxide. $\mathrm{Br} J$ Haematol 1971; 20: 95-111.

34. Bartosz G. Cookbook for novice researchers, reactive oxygen species. In: Second face oxygen: free radical in nature. $2^{\text {nd }}$ ed. Wydawnictwo Naukowe PWN, Warsaw 2003; 376-89.

35. Valle A, O'Connor D, Taylor P, et al. Butyrylcholinesterase: association with the metabolic syndrome and identification of 2 gene loci affecting activity. Clin Chem 2006; 52: 1014-20.
36. Furtado-Alle L, Andrade F, Nunes K, Mikami LR, Souza RL, Chautard-Freire-Maia EA. Association of variants of the -116 site of the butyrylcholinesterase BCHE gene to enzyme activity and body mass index. Chem Biol Int 2008; 175: 115-8.

37. Kutty K, Payne K. Serum pseudocholin-esterase and very-low-density lipoprotein metabolism. J Clin Lab Anal 1994; 8: 247-50.

38. Araoud M, Mhenni H, Hellara I, et al. Plasma cholinesterase activity in hepatic diseases. Ann Biol Clin 2013; 71: 293-8.

39. Montagnese C, Scalfi L, Signorini A, et al. Cholinesterase and other serum liver enzymes in underweight outpatients with eating disorders. Int J Eat Disord 2007; 40: 746-50.

40. Sato K, Hayashi T, Maeda I, et al. Serum butyrylcholinesterase and the risk of future type 2 diabetes: the Kansai Healthcare Study. Clin Endocrinol 2014; 80: 362-7.

41. Iwasaki T, Yoneda M, Nakajima A, Terauchi Y. Serum butyrylcholinesterase is strongly associated with adiposity, the serum lipid profile and insulin resistance. Int Med 2007; 46: 1633-9.

42. Domej W, Földes-Papp Z, Flögel E, Haditsch B. Chronic obstructive pulmonary disease and oxidative stress. Curr Pharm Biotechnol 2006; 7: 117-23.

43. Straface E, Matarrese P, Gambardella L, et al. N-acetylcysteine counteracts erythrocyte alterations occurring in chronic obstructive pulmonary disease. Biochem Biophys Resarch Comm 2000; 279: 552-6.

44. Mercken E, Hageman G, Schols A, et al. Rehabilitation decreases exercise-induced oxidative stress in chronic obstructive pulmonary disease. Am J Respir Crit Care Med 2005; 172: 994-1001.

45. Antczak A, Ciebiada M, Pietras T, Piotrowski W, Kurmanowska Z, Górski P. Exhaled eicosanoids and biomarkers of oxidative stress in exacerbation of chronic obstructive pulmonary disease. Arch Med Sci 2012; 9 : 277-85.

46. Gencer M, Aksoy N, Dagli E, et al. Prolidase activity dysregulation and its correlation with oxidative-antioxidative status in chronic obstructive pulmonary disease. J Clin Lab Anal 2011; 25: 8-13.

47. Ziobro A, Bartosz G. A comparison of the total antioxidant capacity of some human body fluids. Cell Mol Biol Lett 2003; 8: 415-9.

48. Waseem S, Mobarak M, Islam N. Comparative study of pulmonary functions and oxidative stress in smokers and non-smokers. Indian J Physiol Pharmacol 2012; 56: 345-52.

49. Bukowska B, Sicińska P, Pająk A et al. Oxidative stress and damage to erythrocytes in patients with chronic obstructive pulmonary disease - changes in ATPase and acetylcholinesterase activity. Biochem Cell Biol 2015; 93: $574-80$.

50. Nadeem A, Raj H, Chhabra S. Increased oxidative stress and altered levels of antioxidants in chronic obstructive pulmonary disease. Inflammation 2005; 29: 23-32.

51. Gumral N, Naziroglu M, Ongel K, et al. Antioxidant enzymes and melatonin levels in patients with bronchial asthma and chronic obstructive pulmonary disease during stable and exacerbation periods. Cell Biochem Funct 2009; 27: 276-83.

52. Woźniak A, Górecki D, Szpinda M, Mila-Kierzenkowska C, Woźniak B. Oxidant-antioxidant balance in the blood of patients with chronic obstructive pulmonary disease after smoking cessation. Oxid Med Cell Lonegy 2013; 1-9. 
53. Schallreuter K, Elwary S, Gibbons N, Rokos H, Wood J. Activation/deactivation of acetylcholinesterase by $\mathrm{H}_{2} \mathrm{O} 2$ : more evidence for oxidative stress in vitiligo. Biochem Biophys Res Comm 2004; 315: 502-8.

54. Kirkham P, Rahman I. Oxidative stress in asthma and COPD: antioxidants as a therapeutic strategy. Pharmacol Therap 2006; 111: 476-94. 